\title{
Case report: Pathological fracture of the manubrium sternum of unknown aetiology
}

\author{
J Swart, ${ }^{1}(\mathrm{MBChB}, \mathrm{PhD})$; R V P de Villiers, ${ }^{2}(\mathrm{MBChB}, \mathrm{MMed}(\mathrm{Rad}))$; K V Hosking, ${ }^{3}(\mathrm{MBChB}$, FRCS (Ed Ortho)) \\ ${ }^{1}$ Division of Exercise Science and Sports Medicine, Department of Human Biology, University of Cape Town, South Africa \\ ${ }^{2}$ Sports Science Radiology, Winelands Radiology, Sports Science Institute of South Africa, Newlands, South Africa \\ ${ }^{3}$ Cape Orthopaedic Reconstructive Unit, Life Orthopaedic Hospital, Pinelands, South Africa
}

\section{Corresponding author: J Swart (jeroen.swart@uct.ac.za)}

A 26-year-old male professional football player presented with sternal discomfort after performing upper limb exercises. X-rays and a computed tomography (CT) scan of the manubrium sternum indicated an area of lysis with a fracture line. An MRI showed bone marrow oedema as well as presternal and retrosternal soft tissue mass. An incision biopsy of the site and the soft tissue were performed under general anaesthesia. Additional investigations included a bone marrow biopsy from the left iliac crest, a full blood count and C-reactive protein test, all of which were normal. The morphological features were mostly in keeping with a healing fracture site, and the investigations revealed no evidence of a tumour or structural abnormality. In bone injuries, where the level of force is not commensurate with the degree of injury or where the anatomical area and other factors in the presentation are unusual, they require careful investigation. Treating physicians should actively exclude the multitude of potential causes when investigating pathological fractures.

Keywords: pathology, manubrium, stress fracture

S Afr J Sports Med 2017; 29:1-2. DOI: 10.17159/2078-516X/2017/v29i0a3512

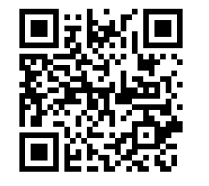

\section{Case report}

A 26-year-old male professional football player (central defender) presented to the Sports Medicine Clinic, Sports Science Institute of South Africa, with a five day history of sternal discomfort. Prior to the onset of pain, the player had been performing tricep dip exercises, during which he suddenly heard an audible click, followed by sternal discomfort.

He had been apparently elbowed during a match four months prior to his presentation at the Sports Medicine Clinic, during which time he experienced pain over the left anterior third and fourth ribs and the left sternal border. Anteroposterior and lateral chest $\mathrm{X}$-rays at that time revealed no abnormality and he returned to play after two weeks without any further symptoms.

He had no relevant medical history, nor was there any family medical history of relevance. His surgical history consisted of a solitary medial meniscal tear of the right knee for which he had had an arthroscopy. He had not been using any medication prior to the onset of pain.

A physical examination revealed tenderness over the junction of the middle and distal third of the manubrium sternum in the midline of the body. There was no palpable mass or irregularity found. Careful examination did not reveal any evidence of cervical, or axillary lymphadenopathy. There was no evidence of goitre or other thyroid enlargement. There were no abnormalities of the respiratory system.

Plain X-rays of the manubrium sternum identified a cortical irregularity of its anterior cortex at the area of maximal tenderness. There was no identifiable fracture line. Due to the minor force involved and the long history between the previous trauma and the new onset of pain, the fracture was assumed to be pathological and further investigations were requested.

\section{CT Scan}

A CT scan was performed (Fig 1 and 2). Sagittal reconstructions showed an area of lysis with a linear fracture line thought to represent a pathological fracture.

\section{MRI Scan}

A magnetic resonance imaging (MRI) scan was also performed. This too demonstrated the fracture line through the manubrium sternum. There was associated bone marrow oedema, as well as an associated presternal and retrosternal soft tissue mass. The differential diagnosis included osteitis (tuberculosis or staphylococcal) or a possible tumour (lymphoma or a round cell tumour).

Blood investigations did not reveal any abnormalities. Specifically, a complete blood count was normal, as were the erythrocyte sedimentation rate and C-reactive protein values.

An incision biopsy of the fracture site and soft tissue were performed under general anaesthesia. A bone marrow biopsy from the left iliac crest was also performed intra-operatively.

\section{Histology}

The frozen section of the biopsy tissue showed trabecular bone between which were spindled cells in keeping with fibrous connective tissue.

Paraffin sections of the biopsy tissue consisted of fragments of trabecular bone. Granulation tissue and fibrous connective tissue were noted within the intervening bone marrow. Osteoid and reactive bone were also noted. Importantly, there was no evidence of lymphoma or other malignancy. The morphological features were in keeping with a healing fracture site.

\section{Bone marrow aspiration}

The bone marrow material showed adequate hemopoiesis in 

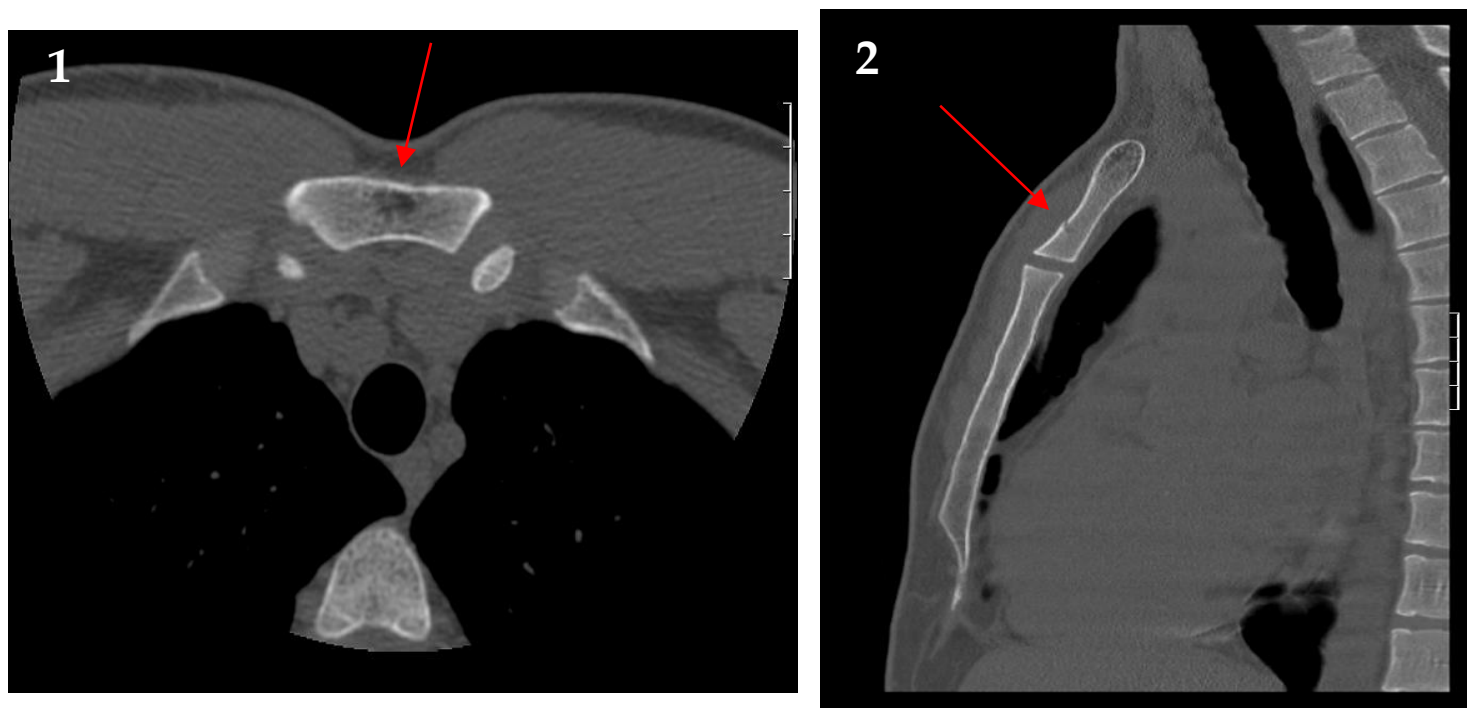

Fig. 1. Axial CT of the sternum, showing a lytic bone lesion fracture.

Fig. 2. CT of the sternum. Sagittal reconstruction shows pathological fracture.

all three hemopoietic cell lines. There were no abnormal infiltrates present in the examined material.

\section{Conclusion}

The investigations revealed no evidence of a tumour or underlying structural abnormality. In the final assessment, it is presumed that the patient sustained a significant bone bruise of his manubrium sternum and subsequently developed a pathological fracture. A stress fracture of the sternum can also not be excluded. Rare cases of stress fractures of the sternum after upper body exercise have been reported. [1,2] At follow-up two weeks after the biopsy, the patient appeared to have improved significantly from a clinical perspective and did not have any discomfort during physical activity or heading (which previously elicited pain). He subsequently made an uneventful recovery and returned to play four weeks post presentation.

Non-traumatic fractures of the sternum are rare. ${ }^{[3-5]}$ The above case, although ultimately benign in outcome, highlights the need for careful investigation of all bone injuries where the level of force is not commensurate with the degree of injury or where the anatomical area and other factors in the presentation are unusual. Treating physicians should actively exclude the multitude of potential causes when investigating pathological fractures.

\section{References}

1. Hill PF, Chatterji S, DeMello WF,et al. Stress fracture of the sternum: an unusual injury? Injury 1997; 28:359-361. [https:// doi.org/10.1016/s00201383(97)00016-8]

2. Robertsen $\mathrm{K}$, Kristensen $\mathrm{O}$, Vejen L. Manubrium sterni stress fracture: an unusual complication of non-contact sport. Br J Sports Med 1996; 30:176177. [https:// doi.org/10.1136/bjsm.30.2.176]

3. Schapira D, Nachtigal A, Scharf Y. Spontaneous fracture of the sternum simulating myocardial infarction. Clin Rheumatol 1995; 14:478-480. [https:// doi.org/10.1007/bf02207687]

4. Aymard A, Chevrot A, Wybier M,et al. Spontaneous fracture of the sternum. J Radiol 1987; 68:593-595. [PMID: 3430442] (French)

5. Swarup S, Bonomally K, Ansari MZ. Fracture of the sternum-an unusual case. Eur J Emerg Med 1999; 6:71-72. [PMID: 10340738] 